\title{
CONTAMINACIÓN ODORÍFERA: CAUSAS, EFECTOS Y POSIBLES SOLUCIONES A UNA CONTAMINACIÓN INVISIBLE
}

\section{ODOUR POLLUTION: CAUSES, EFFECTS AND POSSIBLE SOLUTIONS FOR AN IMPERCEPTIBLE POLLUTION}

\author{
Jaidith Marisol Ramos Rincón ${ }^{1}$ \\ Angye Bermudez ${ }^{2}$ \\ Tania Rojas ${ }^{3}$ \\ ${ }^{1}$ Licenciada en Química, Docente titular Universidad Distrital. \\ 2 Est. Licenciatura en Química, Universidad Distrital. \\ ${ }^{3}$ Est. Licenciatura en Química, Universidad Distrital. \\ 1 marunal2001@yahoo.com \\ 2 anyi_bear@hotmail.com
}

\begin{abstract}
Resumen
La exposición a olores desagradables, se constituye un tipo de contaminación atmosférica proveniente del desarrollo de actividades industriales y humanas que liberan moléculas odoríferas al medio ambiente, que se caracterizan por ser volátiles, lo cual resulta en una calidad del aire no óptimo para el ser humano, afectando en algunas ocasiones su salud; el artículo presenta una revisión bibliográfica acerca de las causas de la contaminación odorífera, sus efectos y sus posibles soluciones; así como las técnicas de análisis, legislación, control y uso, abordado desde el ámbito internacional y nacional.
\end{abstract}

Palabras Clave: Contaminación odorífera, legislación, olor, salud, técnicas de análisis.

\begin{abstract}
Exposure to disgusting smells constitutes a type of atmospheric pollution from industrial and human activities. These activities release volatile odoriferous molecules into the environment that degrade air quality to levels unhealthy to humans. This paper presents a bibliographical review of the causes of odour pollution and its effects and possible mitigation; Aditionally, analyses of legislation, control and use techniques, presented from an international and national regulation perspective.
\end{abstract}

Keywords: Health, legislation, odor, odoriferous pollution, analysis techniques. 


\section{Introducción}

A menudo encontramos noticias, manifestaciones, quejas y demás por los malos olores que emiten las industrias, ríos, lagos, el sector agrícola, la industria de alimentos, las actividades ganaderas, mataderos y actividades de compostaje de origen antropogénico; donde no se encuentra una solución inmediata a esta problemática y por el contrario la población cercana a estas actividades evidencian molestias en la salud; donde a largo plazo afectaría el equilibrio ambiental reflejándose como uno de las tantas causas del calentamiento global. Esta clase de malos olores se puede entender como una sensación resultante de la interacción de especies químicas volátiles inhalados a través de la nariz, incluyendo compuestos de azufre (por ejemplo, sulfuros, mercaptanos), compuestos de nitrógeno (por ejemplo, amoniaco, aminas) y compuestos orgánicos volátiles (por ejemplo, ésteres, ácidos, aldehídos, cetonas, alcoholes) (Leonardos,1969). Estos compuestos que interactúan de manera negativa con el cuerpo humano afectando la calidad de vida, conllevan a poner de manifiesto técnicas efectivas para la remoción de olores que son económicos y aptos para Colombia.

El aumento de este tipo de contaminación es considerado por las autoridades ambientales como un reto complejo de afrontar por el difícil manejo y control de esta contaminación invisible; se hace énfasis en que este tipo de contaminación tiene relación directa con la calidad del aire y por ende con la salud de los ciudadanos en términos de la proliferación de enfermedades respiratorias (IDEAM, 2014).

El presente documento pretende ofrecer una información general acerca de la contaminación odorífera, recopilar algunos estudios en la prevención y control de olores; teniendo como base los aspectos normativos y metodológicos.

\section{Método}

Para esta revisión bibliográfica se consultaron publicaciones indexadas, artículos científicos, revistas científicas y documentos gubernamentales dentro de un rango de fecha de cinco años el cual abarca desde el año 2012 hasta el 2017, los artículos que no entran en este rango son usados por su importancia en aportes a la construcción del presente artículo; la revisión gira en torno a estas cuatro preguntas:

- ¿Qué es la contaminación odorífera?

- ¿Cómo interactúa la contaminación odorífera con el cuerpo humano?

- ¿Cuáles son las causas de la contaminación odorífera?

- ¿Cómo se puede prevenir y controlar la producción de malos olores?

La búsqueda se realiza en las siguientes bases de datos digitales: CABI Forestry Compendium, Ambientalex, CABI Environmental Impact, Springer link, Science direct; Waste Management y Toxnet; revistas científicas: Biblioteca del congreso nacional de Chile, Journal of the Air Pollution Control Association, Revista 100cias@uned, National institute for public health and the environment(RIVM), Acta Neurológica CoIombiana, Journal of Envioronmental Quality, Journal of Environmental Management, Water, Air, \& Soil Pollution, Environmental Science and Pollution Research, American Society of Agricultural and Biological Engineers, Journal of Hazardous Materials, Environmental Research, Front. Environ. Sci. Eng, Chemosphere y documentos de tipo gubernamental del Ministerio de Salud de Colombia, de la Organización Panamericana de la Salud, del Ministerio de Medio Ambiente y Desarrollo Sostenible; entre otros. 
Con base en la consulta del material ya mencionado se llevó a cabo el desarrollo del tema, con los parámetros y objetivos establecidos para esta revisión bibliográfica.

\section{Desarrollo del tema}

Según la norma UNE-EN 13725, el olor se define como "la propiedad organoléptica perceptible por el órgano olfativo cuando inspira sustancias volátiles" (MinSalud - OPS, 2012), por tanto, las moléculas odoríferas son susceptibles de producir un olor que son fáciles de percibir en el olfato que pueden ser agradables o desagradables (García, 2012). La percepción de olor inicia en la emisión de una determinada sustancia o mezcla de sustancias que son transportadas y diluidas en el aire resultando en la inmisión; para luego ser inhaladas por la respiración mediante el sistema olfativo, a través del cual el cerebro genera una respuesta que es la sensación de olor (Bidlingmaier \& Müsken, 2007). Generalmente cuando estos olores generan desagrado, la contaminación odorífera está todavía en imprecisión, porque, aunque bien se ha constituido que se entiende por malos olores y que puede o no afectar a la comunidad en general, los olores no son algo que todas las personas perciban de la misma forma, es algo difuso e inespecífico (MinSalud - OPS, 2012).

Es necesario resaltar algunos conceptos relativos al olor, los cuales se mencionan a continuación: (CONAMA, 2014)

- Olor simple o primario: gracias a la emisión olorosa de un único compuesto determinado, el olfato percibe este de modo aislado.

- Olor compuesto: Se percibe gracias a la mezcla de olores primarios.

- Cantidad de olor: es la mayor o menor intensidad de un olor ya sea simple o compuesto, que es capaz de percibir el olfato humano.
- Concentración de olor: Dimensión utilizada Para caracterizar los olores con fines regulatorios.

- Detectabilidad: Se refiere a la concentración mínima de olor necesario para la detección por un porcentaje específico de la población objeto de investigación, intensidad de olor la que permite determinar la fuerza con la que se percibe la sensación de olor. (Ruijten et al., 2009)

- Umbral del olor: Concentración de olor mínima perceptible por el olfato humano.

- Umbral de percepción de un compuesto: es la concentración mínima de compuesto detectable por el olfato humano, aunque no sea posible su identificación.

- Umbral de identificación: de un compuesto es la concentración mínima de un compuesto con la que es posible su identificación.

- Umbral de exposición: la concentración y el tiempo de exposición al cual se expone un individuo a agentes odoríferos puede ser causa tanto de molestias olfativas como de molestias en la salud, llegando a casos extremos como la muerte.

- Valor límite de exposición a corto término (VLE): valor que no se debe sobrepasar para una exposición máxima de 15 minutos. El respeto de este límite previene los riesgos de efectos tóxicos inmediatos o a corto término.

- Valor límite de exposición media (VME). Se mide para una duración de 8 horas, está destinado a proteger a las personas expuestas a una larga duración. EI VME se puede sobrepasar durante cortos períodos, a condición de no sobrepasar el VLE cuando éste existe (CONAMA, 2014; Rappert \& Muller, 2005).

- Unidad de olor europea: Cantidad de sustancia odorífera que se evapora en $1 \mathrm{~m}^{3}$ en gas neutro en condiciones normales dando una respuesta fisiológica. (García, 2017). 
Teniendo en cuenta estos conceptos relacionados con el olor, el carácter agradable o desagradable de un olor implica una sensación subjetiva, sensación que los humanos y los animales reconocen ante la presencia de químicos contaminantes a través de la estimulación de sus órganos del olfato. Los olores pueden ser agradable (perfume, comida fresca) o desagradable (huevo podrido, aguas residuales). La reacción individual a los olores difiere con la fisiología y aspectos psicológicos. Algunas personas pueden percibir que cierto olor es agradable, mientras que otros pueden percibirlos como desagradables (Nielsen et al, 1998).

Estos compuestos volátiles poseen un peso molecular relativamente bajo. Los compuestos olorosos tienen un valor umbral de olor.

En la gráfica 1 se muestra la cantidad de concentración de partículas odoríferas respecto a la exposición que se da en el ambiente.

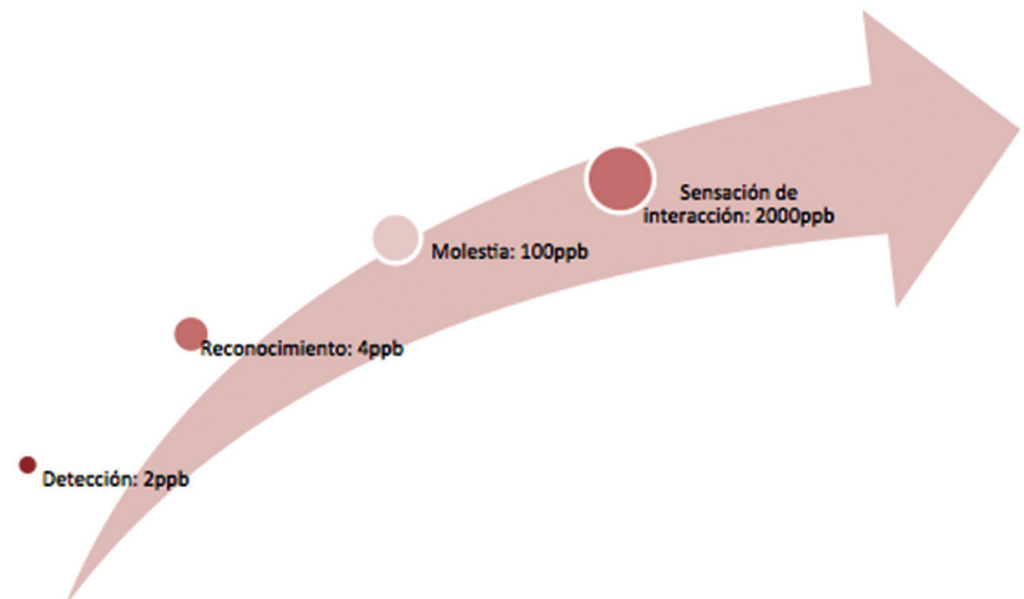

Figura 1. Grado de concentración de la contaminación odorífera.

(Centro de tecnologías limpias de la comunidad Valencia, 2008)

Estas moléculas odoríferas tienen como propiedad que son altamente volátiles por su composición ya que tienen grupos reductores y una elevada polarizabilidad. Aunque dentro estos compuestos odoríferos se encuentran el azufre, el oxígeno y el nitrógeno; que se dan por las explotaciones industriales, agropecuarias, plantas de tratamiento de residuos y estaciones depuradoras de aguas residuales, investigaciones recientes han demostrado que la mayoría de los olores están asociados con los compuestos de azufre volátiles (VSC), especialmente el metanotiol (MT), el sulfuro de dimetilo (DMS) y el disulfuro de dimetilo (DMDS). (Tchobanoglous and Burton, 2003).

A continuación, se da una breve descripción de las sustancias generadoras de la contaminación odorífera, con sus propiedades químicas.

\section{Propiedades químicas asociados a los olores ofensivos:}

Los compuestos descritos a continuación, están asociados con esta contaminación atmosférica.

Sulfuros: Los sulfuros son productos que se descomponen por respiración anaerobia de la materia orgánica y es inflamable que al mezclarse puede ser inflamable y explosivo, pudiendo producir gases tóxicos los cuales pueden ser sulfuro de hidrógeno o ácido sulfhídrico, sulfuro de dimetilo y dicloruro de azufre que pueden estar en un estado gaseoso o líquido (MinSalud - OPS, 2012). 
Mercaptanos: Por otro lado los mercaptanos son tioles que dentro de su estructura contiene azufre que pueden darse en tres ramas como metil, etil y propil mercaptano; estos mercaptanos tienes olores fuertemente repulsivos y por sus tipos de enlaces pueden unirse con las proteínas que se tienen en la piel y producir ciertos olores fétidos algunos de ellos se encuentran como odorantes al gas natural (TOXNET, 09 de abril, 2013; TOXNET, 15 de septiembre, 2005; TOXNET, 27 de junio, 2006)

Gases derivados del nitrógeno: Dentro de esta categoría se encuentran el amoniaco, indol y la metil amina sus características es que son olores penetrantes y característicos del elemento nitrógeno, son malos conductores de energía (TOXNET, 22 de septiembre, 2016; TOXNET, 02 de enero, 2008).

Ácidos orgánicos y derivados: Estos se caracterizan por tener olores picantes, rancios y penetrantes que pueden llegar a ser muy densos y por ser ácidos pueden quemar la piel o donde fue afectado directamente por estos ácidos que aunque puede empezar a ser muy leve puede llegar a causar un gran daño como es el sistema respiratorio; dentro de estos ácidos se encuentran el acetaldehído, ácido butírico y el etil acrilato; compuestos solubles en agua (TOXNET, 14 de septiembre, 2006; TOXNET, 13 mayo, 2010; TOXNET, 14 mayo, 2015).

\section{Compuestos aromáticos y derivados: Es-} tos compuestos son solubles en el agua, pueden a llegar a ser gases muy densos y con la reacción frente a otros químicos pueden liberar olores fuertemente contaminantes y tóxicos, aunque alguno de estos tiene un olor característico dulce puede ser contaminante con el uso de la industria (MinSalud - OPS, 2012). Dentro de estos compuestos se encuentran el benceno, clorofenol y estireno y los COVs, que son hidrocarburos que tienen bajo puntos de ebullición (menor a $100^{\circ} \mathrm{C}$ ) que pueden evaporarse fácilmente o se pueden encontrar a temperatura ambiente (Humane Society International, s.f).

\section{Principales industrias generadoras de contaminación odorífera.}

Los principales compuestos que generan malos olores están relacionados con compuestos azufrados entre otros; donde las actividades que generan este tipo de compuestos naturalmente van a ser foco de contaminación odorífera. Por ello se muestran las actividades que se encuentran como fuentes de contaminación que son:

\section{Explotación de petróleo y gas natural}

En muchos campos petroleros se extrae gas natural que está asociado con el petróleo. En ocasiones el gas natural es utilizado como fuente de energía en las mismas instalaciones o es procesado, y en ocasiones simplemente se quema (acelera el proceso de calentamiento global y afecta a la biodiversidad). Los principales componentes atmosféricos que se producen de la quema de gas son el dióxido de carbono, Metano, Etano, Butano, Propano, Hidrógeno, Helio y Argón, Hidrocarburos Aromáticos Volátiles, Óxido de Nitrógeno, Dióxido de Sulfuro, Ozono, Monóxido de Carbono, Halones, CFCs (Bravo, 2007).

\section{Actividades relacionadas con productos cárnicos}

\section{- Elaboración de productos alimenticios:} en el procesamiento de productos alimenticios se generan olores procedentes de diferentes compuestos en bajas concentraciones, donde no son considerados como amenaza a la salud pública, pero sí una molestia es por esto; que también estas industrias deben funcionar en rigor a las regulaciones debidas. Algunos compuestos 
presentes en esta industria causantes de mal olor son: carbono reducido, nitrógeno y o azufre, aldehídos, cetonas, alcoholes, ácidos, amoniaco, aminas, sulfuros, entre otros; estos también pueden ser derivados de la descomposición de alimentos (Rappert \& Muller, 2005).

\section{- Tratamiento de cuero o curtiembres:} estas industrias están catalogadas como unas de las más sucias y contaminantes, el proceso que se lleva a cabo allí comienza teniendo el cuero crudo seguido del procesamiento del cuero para darle el acabado, la manufactura y así; de esta forma comercializar el producto, los malos olores que provienen de estas industrias están estrechamente ligados a la falta de control en las aguas residuales las cuales presentan altas concentraciones de materia orgánica provocando condiciones anaerobias en los cursos de agua, esto dado que se presenta un consumo elevado del oxígeno disuelto; además los residuos sólidos provienen en su mayoría del proceso de descarnado. En el proceso de pelambre se requiere el uso de cal y sulfuro de sodio (aporta la mayor cantidad de olores desagradables), no solo en esta etapa si no que en las demás etapas (descarne, control deficiente en la limpieza de equipos, vertimiento de efluentes líquidos y sólidos en las corrientes de agua) relacionadas con estas industrias, ayudan a que cada día la afectación sea mayor, a los alrededores donde se sitúan las curtiembres es inevitable que el número de quejas sea mayor diariamente, afectando la calidad de vida de estas comunidades y generando alteraciones en la salud, todo debido a la fuerte emanación de olores. (MinSalud - OPS, 2012; CONAMA, 2012).
- Fabricación de abonos y compuestos orgánicos: Algo importante es tener en cuenta que en la ciudad se llevan cabo actividades que son propias de campos abiertos y necesitan un buen manejo de desechos, por ejemplo, funcionamiento de galpones de aves en la ciudad, esto genera mucho malestar por los olores que se desprenden.

- Las operaciones agrícolas, la práctica de ganadería intensiva, los problemas asociados con el tratamiento de los desperdicios, la ventilación de las edificaciones donde se llevan a cabo estas prácticas las cuales despiden todo tipo de malos olores relacionados con el estiércol y los biosolidos, sin contar con la distribución de fertilizantes agrícolas que acrecientan la contaminación en suelos y aguas (Rappert \& Muller, 2005). Este tipo de contaminación es una problemática difícil de manejar, esto dado que es producido por varias fuentes, las cuales se deben controlar de diferente forma, esto también se ve afectado porque no solo se verá un episodio constante de olor si no que este variará de tal forma que los efectos producidos estarán relacionados con daños tanto físicos como mentales, teniendo en cuenta que un periodo expuesto a molestias por olor se relaciona con dolores de cabeza, molestias al dormir, ganas de vomitar, pérdida del deseo de comer etc. (Canales, Borquez, \& Vega, 2007).

A continuación, se muestra en la tabla 1 una relación entre los principales compuestos generadores de malos olores y las industrias productoras de estos. 
Tabla 1. Industrias productoras de malos olores. Tomado de (Baena \& Hernández, s.f)

\begin{tabular}{|c|c|}
\hline Componente Oloroso & Actividades industriales que los generan \\
\hline Sulfura de hidrogeno & $\begin{array}{l}\text { Papeleras, refinerías, industrias y actividades agrarias, } \\
\text { vertederos, depuradoras. }\end{array}$ \\
\hline Mercapatanos & $\begin{array}{l}\text { Papeleras, refinerías, industrias y actividades agrarias, } \\
\text { vertederos, depuradoras. }\end{array}$ \\
\hline $\begin{array}{l}\text { Sulfuro de metilo } \\
\text { y sulfuro de dimetilo }\end{array}$ & Papeleras. \\
\hline Amoníaco & $\begin{array}{l}\text { Refinerías, fábricas de fertilizantes, industrias y } \\
\text { actividades agrarias, vertederos y depuradoras }\end{array}$ \\
\hline Dióxido de azufre & Refinerías, centrales térmicas, calderas. \\
\hline Óxidos de nitrógeno Y Urea & Fábricas de fertilizantes. \\
\hline Aldehídos & Fábricas de fertilizantes, tratamientos de metales. \\
\hline Aminas & Industrias agroalimentarias, vertederos, depuradoras \\
\hline
\end{tabular}

\section{Efectos en la salud humana}

Según la OMS un mal efecto en la salud es cualquier experiencia que provoque malestar a nivel, tanto físico como emocional o mental, desde este punto de vista sin duda la contaminación odorífera genera molestias en la salud, las personas que se encuentran cercanas al desarrollo de actividades generadoras de malos olores, padecen insomnio, mal humor, dolor de cabeza, irritación en mucosa, sobre todo la tendencia a desarrollar situaciones de estrés, (Valencia, 2008) náuseas, vómitos, reacciones aparentemente neurotóxicas; tales como comportamiento evasivo, pérdidas de memoria o problemas de concentración, interacciones con otros sistemas sensoriales o biológicos que provocan cambios de hipersensibilidad y cambios en las pautas de respiración (Subils, s.f). Estos efectos se dan porque al ser captadas las sustancias odoríferas del medio ambiente por vía nasal o bucal transportadas a la mucosa olfatoria, donde se reciben y posteriormente se traducen en señales eléctricas; (Cantillo, 2016) el tracto respiratorio incluye una reducción del volumen del aire inhalado, contracción de la laringe y los bronquios; mayor secreción de hormonas de estrés, presión sanguínea elevada o un flujo sanguíneo menor en los pulmones (Humane Society International, s.f).

Los olores son señal del problema que producen respuestas en las personas, ya que actúan sobre el sistema nervioso central o periférico (Gómez, 2015) y están relacionados sobre todo con la parte emocional, con los recuerdos y el humor. Es por esto que un olor agradable puede producir sensación de bienestar, y un mal olor puede producir dolor de cabeza, en ocasiones, náuseas ronquera y por supuesto una alteración en el estado de ánimo (Schiffman, 2005). Estas molestias como se mencionó anteriormente que son efectos negativos físicos y mentales se ha demostrado que si bien los malos olores no producen la muerte si producen graves daños, como la pérdida del sentido del olfato limitando a la persona a una vida sin olores, sin algunos recuerdos y en muchos casos sin sabores entre otras consecuencias de gran preocupación, (Gómez, 2015) puesto que el olfato y el gusto son complementarios. En la figura 2 se muestra una relación entre causas, efectos y legislación de la contaminación odorífera. 


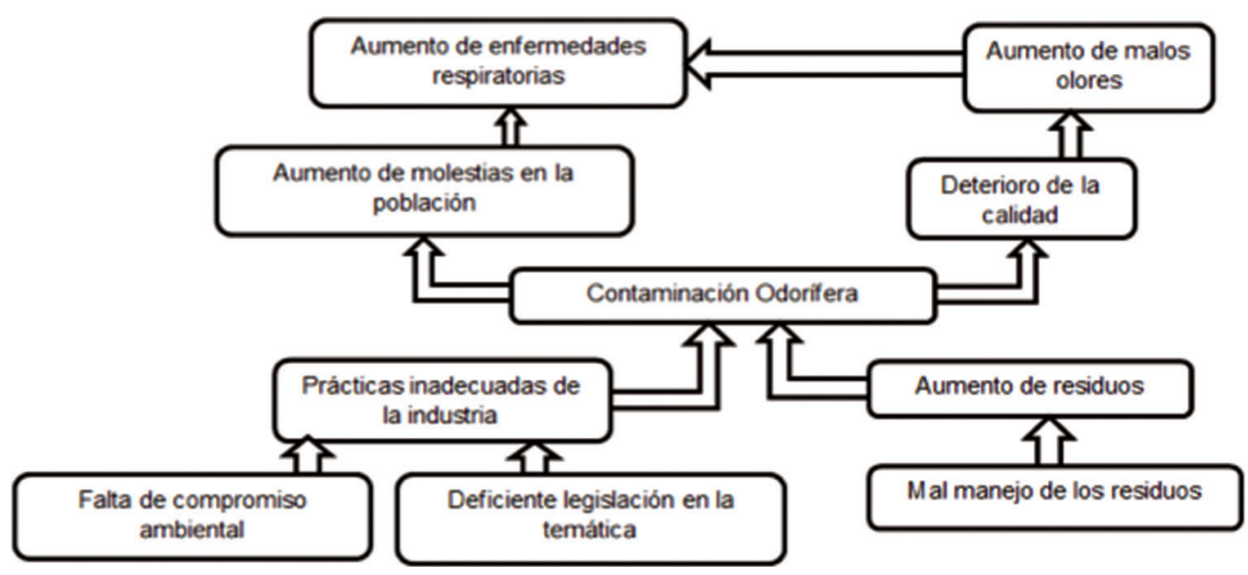

Figura 2. Problemática contaminación odorífera. (Elaboración propia)

Técnicas de análisis de olores:

La contaminación odorífera, es realmente algo problemático, ya que el umbral de olor para todas las sustancias no es el mismo, además nuestro órgano olfativo tiene una gran sensibilidad, y puede percibir de manera exagerada la concentración de alguna sustancia presente en el aire; por supuesto se sabe que es poco confiable fiarnos de nuestros sentidos como un patrón de medida, es por esto que se han desarrollado tecnologías que pueden medir con un poco más de precisión y exactitud la concentración de la sustancia que está generando malestar (Asociación Española para la calidad, s.f). A continuación se describen diferentes técnicas de análisis de olores:

Existen algunas estrategias de análisis de olores las cuales están enfocadas adecuadamente donde se usan técnicas de tipo sensorial y de tipo fisicoquímico. Hay métodos directos, sensoriales u olfatométricos que involucran el uso de la nariz humana a cargo de un grupo de observadores, este método se subdivide en dos categorías: escalamiento y dilución; la primera califica un olor dentro de una clasificación de intensidad y la segunda realiza una dilución en una corriente de aire libre de olores con el fin de determinar el umbral de olor (Rappert \& Muller, 2005).
También existe la olfatometría dinámica la cual es una técnica sensorial, que se lleva a cabo gracias a la intervención de personal experto (olfatómetras), mediante la utilización de un olfatómetro; este aparato funciona de tal manera que un gas neutro sirve para diluir la muestra de gas oloroso hasta una concentración que coincida con el umbral de detección, el cual se establece cuando el $50 \%$ de las personas (olfatómetras) perciben el olor de la muestra diluida (Xarxa ambiental, s.f).

Las narices electrónicas tienen unos sensores altamente sofisticados, que tienen la capacidad de captar las sustancias olorosas y relacionarlas con unos patrones ya registrados en sus bases de datos, dar una respuesta muy aproximada de que clase de sustancia produce el mal olor y en que concentración está presente en el medio; estas narices con sus sensores actúan como un entramado de nervios que al relacionar las diferentes evaluaciones de cada sensor responde acerca de lo que ha captado y analizado (García, 2012).

Otros instrumentos para evaluar los malos olores son: tablas FIDO, mediciones de campo, nomogramas, quejas, modelización; estas herramientas son estudios que se hacen directamente con la población afectada, buscando 
encuestar para determinar el tiempo en el cual se presenta el mal olor, que tipo de olor, de que manera les afecta y si es realmente desagradable y/o fuente de enfermedades (Asociación Española para la calidad, s.f).

Los métodos indirectos son métodos instrumentales como por ejemplo la cromatografía de gases; estas técnicas dan razón de las concentraciones de los compuestos presentes en el aire, los cuales son identificados al ser comparados con información de sus bases de datos. Los métodos analíticos tradicionales se han utilizado para la detección de compuestos productores de olor, utilizando una combinación de la cromatografía de gases (GC) con un cierto enfoque de detección, como la detección de ionización de llama (GC / FID), fotoionización (GC / PID) o espectrometría de masas (GC / MS) (H. Skov, A. Lindskog, F. Palmgren and C. S. Christensen,(2001), X.-L. Cao and C. N. Hewitt, (1995)).

Los métodos actuales como GC-MS, (CONAMA, s.f) PID (El detector de fotoionización), IPD (detector fotoacústico infrarrojo) entre otros; permite tener información de la composición real de los gases en el aire, pero no de la percepción humana del mismo.

A partir de estos instrumentos se puede acoplar a un modelo matemático donde calcula las emisiones de olores, su composición, impacto del olor entre otras. Este modelo es aplicado en vertederos para así conocer todo lo mencionado anteriormente, localizar los más contaminantes y así dar solución a esta polución en el aire; llamado modelo de dispersión gaussiana (Bofeng, 2015).

\section{Remoción de los contaminantes de una emisión.}

El proceso de remoción o eliminación de malos olores depende directamente del estado del contaminante; ya sea gaseoso (se remueven por difusión hacia superficies controlables, en donde se pueden realizar reacciones químicas para transformar estos gases en productos menos nocivos) o en forma de partículas. Para llevar a cabo estos procesos existen medios de control sin dispositivos (cambio de proceso, cambio de combustible, buenas prácticas de operación, cierre de la planta); y medios de control con dispositivos (incineradores termales, absorbedores de carbón, absorbedores, condensadores, precipitadores electrostáticos, filtros, cámaras de sedimentación, ciclones) (Inche, 2004).

En el plan nacional de calidad del aire del ecuador se presentan tres programas (control y vigilancia de calidad del aire; mejoramiento de la calidad del aire y prevención de su deterioro y medidas a ser aplicadas durante los estados de alerta), estos presentan sus respectivos proyectos donde se resalta el segundo programa, ( Competencias sobre la gestión del recurso aire, Formación, capacitación e investigación en gestión de la calidad del aire integrados a las políticas nacionales de ciencia y tecnología, Implantación del programa nacional de reducción de emisiones, Producción de combustibles de mejor calidad para fuentes móviles y fijas, Sistema nacional de revisión técnica vehicular, Sistema de participación ciudadana en tema calidad del aire), que busca involucrar a todas las comunidades para que sean parte del cambio (Ministerio del Ambiente, s.f).

En cuanto a técnicas de desodorización adecuadas aparecieron; el lavado químico, adsorción y procesos biológicos (Anet, 2013). En la actualidad existen técnicas diversas para la desodorificación del aire, dentro de las cuales encontramos:

- Tratamiento por biofiltración: este tratamiento es un proceso ecológico, económico y robusto (Anet, 2013); estos biofiltros 
tienen una capa colonizada con microorganismos capaces de degradar los malos olores provenientes de las aguas, la biomasa, el $\mathrm{CO}_{2}$, entre otros (Anet, 2013). Estos biofiltros que son de bajo costo son eficaces, sin embargo el rendimiento del biofiltro no es uniforme, y está influenciado por varios parámetros de diseño y operación, tal como composición de gas, material de empaquetado, humedad, temperatura, $\mathrm{pH}$, caída de presión y residencia de gas (Vieira, 2016); ésta técnica es aceptada por ser una tecnología eficiente y limpia, la cual no utiliza sustancias químicas, evitando así la generación de residuos peligrosos difíciles de ser tratados (Gutiérrez, 2008); dentro de los materiales de bajo costo usados para biofiltración se pueden mencionar los siguientes: la fibra de coco, turba fibrosa, brezo, turba y brezo, turba granulada, astillas de madera, barks, abono compostado, carbón activado, esquisto expandido, pouzzolane, siendo el material más eficiente y viable las astillas de madera en aspectos económicos, físicoquímicos y biológicos (Anet, 2013).

- También se encuentra un biofiltro móvil diseñado en dos etapas una con material de cedro y la otra parte con astillas de madera; material que se encuentran en cualquier país, estos tienen la capacidad de almacenamiento de agua, porosidad entre otros aspectos físicos; llamados chips WC; estos muestran que reducen el $51 \%$, $83 \%$ y $41 \%$; el olor, el $\mathrm{H}_{2} \mathrm{~S}$ y el $\mathrm{NH}_{3}$, respectivamente (Chen, 2012).

- Fotocatalizadores: este tratamiento ha sido validado como una tecnología prometedora para la eliminación de compuestos orgánicos volátiles odoríferos tales como: $\mathrm{H}_{2} \mathrm{~S}$, mezcla de compuestos orgánicos volátiles (butanona, tolueno y a-pineno), tricloroetileno, mezcla de tolueno, mezcla de etanotiol, disulfuro de dimetilo y tioanisol; con biofiltros y percoladores (BFT) que se combinan con fotocatalizadores; ya que existen sustancias que no pueden ser degradadas de forma biológica, esta integración produce la oxidación de compuestos que posteriormente son fácilmente biodegradables (Lia, 2013).

- Tecnología de Fitorremediación: este tratamiento es usado para la eliminación de olores de trietilamina, presentes en el pescado, aguas residuales, residuos de vertedero, cría de ganado, estiércol de cerdo e instalaciones de compostaje y eliminación de compuestos orgánicos volátiles (COV) tales como BTEX (benceno, tolueno, etilbenceno y xileno), amoniaco, formaldehído, etc; por las plantas crasuláceas (CAM metabolismo ácido de las crasuláceas) y Pterocarpus indicus (C3) (Phattara, 2015).

- Tratamiento de enmascaramiento: Esta técnica consistes en usar otro componente odorífero de olor agradable al ser humano para así enmascarar el olor desagradable; (Grupo PPA, s.f) aunque a menudo no causa contaminantes secundarios; no resuelve las emisiones de contaminantes en el aire utilizándose solo en caso de emergencia (Lewkowska, 2016).

- Tratamiento por aditivo microbiano mineral: Este tratamiento esta dado para la reducción de amoniaco, aminas y $\mathrm{H}_{2} \mathrm{~S}$ de las emisiones de estiércol de vaca, este aditivo microbiano está compuesto por polvo mineral que es una perlita-bentonita y polvo de bacterias; pero siendo este tratamiento muy costoso por el tiempo, química a utilizar y que solamente sirve para la eliminación de estos olores (Kajetan, 2017).

\section{Marco legal}

Dentro de la gráfica 3 se logra apreciar los parámetros FIDO, los cuales proporcionan 
criterios para comprender el marco legal a nivel mundial en Colombia (tabla 2) y realizar una evaluación en cuanto a los enfoques relacionados a la contaminación odorífera.

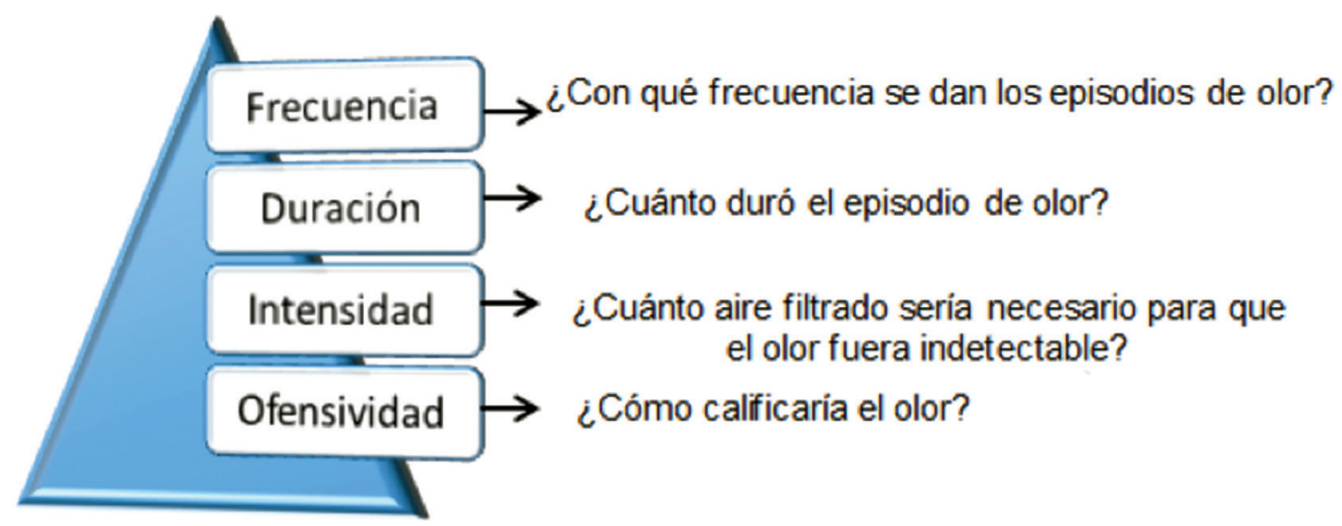

Figura 3. Evaluación de molestias por malos olores.

Fuente: (Kajetan S, 2017).

Tabla 2. Ejemplos del marco legal con el FIDOL.

\begin{tabular}{|c|c|c|}
\hline Enfoque & Descripción & Jurisdicción \\
\hline \multirow{2}{*}{$\begin{array}{l}\text { estándar } \\
\text { máximo } \\
\text { impacto }\end{array}$} & Olor & $\begin{array}{l}\text { jurisdicciones de Canadá, Chile, Colombia, jurisdicciones de Estados Unidos, } \\
\text { Panamá, Paraná (Brasil), Reino Unido, Alemania, Austria, Lombardía (Italia), } \\
\text { Puglia (Italia), Irlanda, Países Bajos, Israel, Taiwán, Hong Kong, Corea del Sur, } \\
\text { China, Japón , las jurisdicciones de Australia, Hungría, las jurisdicciones belgas, } \\
\text { Cataluña (España), Dinamarca, las jurisdicciones belgas, Francia, Austria }\end{array}$ \\
\hline & $\begin{array}{l}\text { productos químicos } \\
\text { individuales } \\
\text { relacionados olor }\end{array}$ & $\begin{array}{l}\text { jurisdicciones de Canadá, Colombia, jurisdicciones de Estados Unidos, Sao } \\
\text { Paulo (Brasil), Panamá, Corea del Sur, Japón, las jurisdicciones australianas, } \\
\text { las jurisdicciones de Australia, Dinamarca, Puglia (Italia) }\end{array}$ \\
\hline \multirow{2}{*}{$\begin{array}{l}\text { estándar de } \\
\text { distancia de } \\
\text { separación }\end{array}$} & Variable & $\begin{array}{l}\text { jurisdicciones de Estados Unidos, Paraná (Brasil), Austria, Países Bajos, } \\
\text { las jurisdicciones australianas, las jurisdicciones belgas, Dinamarca, las } \\
\text { jurisdicciones belgas, las jurisdicciones de Canadá, Austria, Alemania }\end{array}$ \\
\hline & Fijo & $\begin{array}{l}\text { jurisdicciones canadienses, jurisdicciones de Estados Unidos, Holanda, Hong } \\
\text { Kong, jurisdicciones de Australia, Alemania }\end{array}$ \\
\hline \multirow{2}{*}{$\begin{array}{l}\text { norma de } \\
\text { emisión } \\
\text { máximo }\end{array}$} & Olor & Francia, jurisdicciones italianas, China, las jurisdicciones de Australia, Dinamarca \\
\hline & $\begin{array}{l}\text { productos químicos } \\
\text { individuales } \\
\text { relacionados olor }\end{array}$ & $\begin{array}{l}\text { Chile, Panamá, Brasil (federal), Puglia (Italia), China, Japón, las } \\
\text { jurisdicciones australianas }\end{array}$ \\
\hline \multirow{2}{*}{$\begin{array}{l}\text { estándar } \\
\text { molestia } \\
\text { máximo }\end{array}$} & Número de quejas & jurisdicciones de Estados Unidos, Wellington (Nueva Zelanda) \\
\hline & nivel de molestia & Nueva Zelanda \\
\hline $\begin{array}{l}\text { estándar de } \\
\text { la tecnología }\end{array}$ & MURCIÉLAGO & $\begin{array}{l}\text { países europeos, las jurisdicciones canadienses, jurisdicciones de Estados } \\
\text { Unidos, las jurisdicciones de Australia, Nueva Zelanda, Arabia Saudí, Colombia }\end{array}$ \\
\hline
\end{tabular}

Tomado de Brancher (2017) 
Dentro de esta tabla se logra apreciar que Colombia hace parte de diferentes enfoques respecto a sus avances tecnológicos y científicos, aunque no tiene jurisdicción para la emisión máxima de olor, estándar de molestia máximo y estándar de distancia de separación, que son necesarias para la mayor regulación en las actividades antropogénicas donde sus productos son la principal causa de enfermedades en la salud humana, se debe empezar a mejorar estos tipos de estándares que son los más importantes en la industria Colombiana y mejorar el marco legal, ya que este tipo de contaminación es invisible ante la sociedad.

Para realizar una queja por olores ofensivos se tienen en cuenta dos aspectos o condiciones; debe existir una fuente generadora de olores y debe haber un receptor o indicador de la presencia de olores ofensivos, que en este caso es el que presenta una queja formal.

La presentación de la queja es fundamental para el tratamiento de la problemática, una vez exista una queja, se evalúa; el procedimiento de evaluación se realiza con base en encuestas estandarizadas en la norma técnica NTC 601211 "Efectos y evaluación de los olores. Evaluación sicométrica de las molestias por olores. Cuestionarios"; en continuidad a ello las autoridades ambientales competentes realiza una planeación para la reducción de los olores ofensivos, posteriormente se realiza una medición con el fin de verificar una reducción del impacto ambiental por olores ofensivos.

La NTC 6012-1 es la norma técnica colombiana donde se describen los métodos de investigación ya estandarizados para determinar el potencial de molestia que pueden causar los olores ofensivos, como ya se mencionó es importante que la comunidad en general se entere que las entidades gubernamentales tienen herramientas para solucionar las molestias que puedan sentir causadas por olores desagradables, pero que para esto el receptor, que en este caso son los ciudadanos afectados, deben quejarse para que los entes encargados y capacitados en este oficio puedan evaluar la situación. Teniendo en cuenta lo anterior la NTC 6012-1 establece que posterior a la queja se deberá establecer el tipo de método, este puede ser directo, indirecto o por criterios de aceptación o tolerancia, después deberá planificar una encuesta y medir la molestia, esta molestia se mide seleccionando el área, recolectando datos los cuales incluyen tomas de muestras, contacto con los encuestados, análisis de laboratorio, reportes, entre otros; los reportes deben ser analizados y comparados con base en las cantidades permitidas de sustancias en el ambiente, las cuales ya están fijadas en la norma, la puntualidad de cada procedimiento se encuentra en la NTC 6012-1, si se requiere más información de los métodos y procedimientos (Ministerio de ambiente y desarrollo sostenible, 2014).

\section{Conclusiones}

La contaminación odorífera constituye una problemática seria en salud pública, aunque no se vea de esta manera; en diferentes fuentes se ha encontrado que los olores ofensivos sí generan enfermedades a las personas tales como irritación de garganta, pérdida del apetito, náuseas, dolor de cabeza, estados de ánimo afectados, etc.; teniendo en cuenta lo anterior, es de considerarse como una situación por la cual el estado debe preocuparse y velar, atender las quejas y buscar soluciones prontas a las inconformidades de la o las comunidades afectadas, no es sano ni agradable vivir constantemente respirando un aire de mala calidad, en condiciones tal vez de insalubridad y molestia; es por esto que las personas deben enterarse de la normativa que rige el funcionamiento de las industrias y el desarrollo de actividades generadoras de 
desechos y de malos olores, y de la normativa que existe en pro del bienestar de los ciudadanos comunes, y mejorar su calidad de vida entendiendo que es deber de las autoridades ambientales velar por la salud física y mental de cada uno de los ciudadanos que se ven amenazados por este tipo de contaminación. En la actualidad existe tecnología muy sofisticada para la detección de malos olores y sobre todo existe la obligación del estudio a fondo de la problemática, en este momento no hay razón para pasar por alto anomalías ambientales de este tipo, que bien pueden empezar con el síntoma de un olor ofensivo pero que a profundidad pueden evitar problemas que amenacen la salud y los recursos naturales. Se plantean diferentes métodos de remoción de olores que son económicos, aplicables en las industrias generadoras de olores y viables en Colombia, por ejemplo, el tratamiento por biofiltración, fotocatalizadores entre otros. A pesar que los factores FIDOL dentro de su gran mayoría se aplican en Colombia, se encuentra un vacío para las normas de emisión máximo y estándar de separación que son importantes y unas de las mayormente a considerar ya que siendo una contaminación invisible podemos estar en un alto rango de contaminación; aunque el marco legal de nuestro país que contempla el tratamiento de olores ofensivos es útil y claro es posible mejorarlo y aplicarlo en todas las situaciones que sea necesario, teniendo como parámetro otros lugares del mundo que han desarrollado proyectos muy interesantes con muy buenos resultados; todo esto con el fin de brindar una mejor calidad de vida a todos los ciudadanos de nuestro país; también dentro de este artículo se proporciona al ciudadano conocimiento acerca de los olores que se pueden percibir y las industrias generadoras de ello para poner un alto a una contaminación tan grave $y$ perjudicial para la vida humana.

\section{Agradecimientos}

Agradecemos a la Universidad Distrital Francisco José de Caldas por el aval y respaldo en esta producción académica y en especial a la profesora Marisol Ramos por su constante apoyo y paciencia durante este proceso.

\section{Literatura citada}

Asociación Española para la Calidad. (s.f). Contaminaciónodorífera. Recuperado dehttps://www.aec. es/c/document_library/get_file?uuid=3527643c0525-42fd-9943-1c881254e44f\&groupId $=10128$

Baena, S. \& Hernández, L. (s.f). Análisis de la regulación colombiana en materia de olores ofensivos. Recuperado de http://repository.udem.edu.co:8080/bitstream/handle/11407/1154/An\%C3\%A1lisis\%20de\%20 la\%20regulaci\%C3\%B3n\%20colombiana\%20 en\%20materia\%20de\%20olores\%20ofensivos. pdf?sequence $=1 \&$ is Allowed $=y$

Benoît, A. (2013). Characterization and Selection of Packing Materials for Biofiltration of Rendering Odourous Emissions. Water, Air, \& Soil PoIlution, 1-13.

Benoît, A. (2013). Characterization of gaseous odorous emissions from a rendering plant by GC/MS and treatment by biofiltration. Journal of Environmental Management, 981-987.

Bidlingmaier, W. \& Müsken, J. (2007). Chapter 11 Odor emissions from composting plants. Waste Management Series Elsevier., Volumen 8 215-324.

Bofeng, C. (2015). Evaluating the impact of odors from the 1955 landfills in China using a bottomup approach. Journal of Environmental Management, 206-214.

Brancher, M. (2017). A review of odour impact criteria in selected countries around the world. Chemosphere, 1531-1570.

Bravo, E. (mayo, 2007). Los impactos de la explotación petrolera en ecosistemas tropicales y la biodiversidad. Recuperado de https://www.inredh.org/archivos/documentos_ambiental/impactos_explotacion_petrolera_esp.pdf

Canales, P., Borquez, B., \& Vega, J. (2007). La contaminación por olores y su regulación en la legislación nacional y extranjera (España y Alemania). Biblioteca del congreso nacional de Chile. 
Cantillo, G. (2016). Disfunción olfatoria pos-trauma encéfalocraneano y su impacto en la calidad de vida: revisión de tema. Acta Neurológica Colombiana, 161-168.

Centro de tecnologías limpias de la comunidad Valencia. (2008). Guía técnica para la gestión de emisiones odoríferas. España.

CONAMA (2012). Contaminación odorífera, documento del grupo de trabajo de Conama. Recuperado de http://www.conama11.vsf.es/conama10/download/files/conama11/GTs\%202010/6_final.pdf.

CONAMA (2014). Contaminación odorífera. Recuperado de http://www.malosolores.org/pdf/propia/ GT-11_doc_preliminar.pdf

CONAMA. (s.f). Contaminación odorífera. Estrategias de análisis de olores.

Chen, S. (2012). A Two-Stage Wood Chip-Based Biofilter System to Mitigate Odors from a DeepPit Swine Building. American Society of Agricultural and Biological Engineers, 893-901.

García, A. I. (2012). Contaminación odorífera. Estrategias de análisis de olores. Revista 100cias@ uned, 92-100.

García, A. R. (2017). Contaminación atmosférica por olores: unas técnicas de medida avanzadas y una legislación específica inexistente. Recuperado de http://blog.condorchem.com/pdf/ponencia_olors_conama9.pdf

Gómez, E. (2015). Olor y derecho. San Vicente del Raspeig: Universidad de Alicante.

Gutiérrez, A. (2008). Efecto de la carga másica y altura del medio filtrante sobre la remoción de h2s mediante biofiltración. Mexico: Universidad Nacional Autónoma de México.

H. Skov, A. Lindskog, F. Palmgren and C. S. Christensen,(2001). An overview of ommonly used methods for measuring benzene in ambient air, Atmospheric Environment 35 141-148.

Humane Society International. (s.f). Los impactos sobre la salud humana de olores emitidos por instalaciones de cría intensiva de animales de producción. Recuperado de http://www.hsi.org/assets/pdfs/hsi-fa-white-papers/odors-spanish.pdf

Inche, J. (2004). Gestión de la calidad del aire: Causas, efectos y soluciones. Lima: Instituto de Investigación de Ingeniería Industrial-UNMSM, 89-92.
Instituto de Hidrología, Meteorología y Estudios Ambientales IDEAM. (2014). Calidad del aire. Recuperado de http://www.ideam.gov.co/web/contaminacion-y-calidad-ambiental/calidad-del-aire

Kajetan, S. (2017). Odour reducing microbial-mineral additive for poultry manure treatment. Front. Environ. Sci. Eng, 1-9.

Leonardos, G. (1969). Odor threshold determinations of 53 odorant chemicals. Air Pollut. Control Assoc. 19, 91-95.

Lewkowska, P. (2016). Characteristics of odors emitted from municipal wastewater treatment plant and methods for their identification and deodorization techniques. Environmental Research, 573-586.

Lia, Z. (2013). Pollution profiles, health risk of VOCs and biohazards emitted from municipal solid waste transfer station and elimination by an integrated biological-photocatalytic flow system: A pilot-scale investigation. Journal of Hazardous Materials, 147-154.

Magnun, M. \& Vieira, W. (2016). Pragmatic evaluation of odour emissions from a rendering plant. Environmental Science and Pollution Research, 115-124.

Ministerio de ambiente y desarrollo sostenible. (2014). Protocolo para el monitoreo, control y vigilancia de olores ofensivos. Recuperado de http://www.minambiente.gov.co/images/ Atencion_y_particpacion_al_ciudadano/Consulta_Publica/Protocolo_para_el_Monitoreo_ Control_y_Vigilancia_de_Olores_Ofensiv.pdf

Ministerio de medio ambiente y desarrollo sostenible. (12 noviembre, de 2013). Resolución $N^{\circ}$ 1541. Recuperado de http://www.boyaca.gov. co/SecSalud/images/Documentos/Salud_Publica/Ano_2014/Residuos-solidos/resolucion $\% 20$ 1541\%20de\%202013\%20-\%20Olores.pdf

Ministerio de salud y protección social y Organización panamericana de la salud. (2012). Lineamientos para la vigilancia sanitaria y ambiental de los olores ofensivos en la salud y calidad de vida de las comunidades expuestas en áreas urbanas. Recuperado de https://www.minsalud. gov.co/sites/rid/Lists/BibliotecaDigital/RIDE/VS/ $\mathrm{PP} / \mathrm{SA}$ /impacto-olores-ofensivos-salud.pdf

Ministerio del Ambiente. (s.f). Plan nacional de la calidad del aire. Recuperado de http://www. ambiente.gob.ec/wp-content/uploads/downloads/2012/10/libro-calidad-aire-1-final.pdf 
Nielsen, P.H., Raunkjaer, K., Hvitved-Jacobsen, T.H., (1998). Sulfide production and wastewater quality in pressure mains. Water Sci. Technol. $37,97-104$.

Phattara, P. (2015). Removal of trimethylamine (fishy odor) by $\mathrm{C} 3$ and CAM plants. Environmental Science and Pollution Research, 543-557.

Productos plásticos anticorrosivos Grupo PPA. (s.f). Instalaciones de desodorización ambiental. Recuperado de http://grupoppa.es/pdf/INST\%20 DESODORIZACIONES.pdf

Rappert, S. \& Muller, R. (2005). Odor compounds in waste gas emissions from agricultural operations and food industries. Waste Management, 887-907.

Ruijten, M. Van Doorn, R. \& Van Harreveld, A. (2009). Assessment of Odour Annoyance in Chemical Emergency Management. National Institute for Public Health and Environment.

Schiffman, M. (2005). Science of odor as a potential health issue. Journal of Envioronmental Quality, 129-138.

Subils, J. (s.f). NTP 358: Olores: un factor de calidad $y$ confort en ambientes interiores. Recuperado de http://www.insht.es/InshtWeb/Contenidos/Documentacion/FichasTecnicas/NTP/ Ficheros/301a400/ntp_358.pdf

Tchobanoglous, G., Burton, F.L., (2003). Wastewater Engineering Treatment and Reuse, fourth ed. Tata McGraw-Hill, New Delhi.

TOXNET (14 febrero, 2003). Indole. [Base de datos]. Recuperado de https://toxnet.nlm.nih.gov/ cgi-bin/sis/search2/f?./temp/ 6UFNs4:1

TOXNET (15 de septiembre, 2005). Ethyl mercaptan. [Base de datos]. Recuperado de https:// toxnet.nlm.nih.gov/cgi-bin/sis/search2/f?./ temp/ Eyv42F:1

TOXNET (14 de septiembre, 2006). Butyric acid. [Base de datos]. Recuperado de https://toxnet.nIm.nih.gov/cgi-bin/sis/search2/f?./ temp/ thzMJd:1

TOXNET (27 de junio, 2006). Propyl mercaptan. [Base de datos]. Recuperado de https:// toxnet.nlm.nih.gov/cgi-bin/sis/search2/f?./ temp/ LRZrcN:1

TOXNET (02 de enero, 2008). Methylamine. [Base de datos]. Recuperado de https://toxnet.nlm.nih. gov/cgi-bin/sis/search2/f?./temp/ 7f9msO:3
TOXNET (21 de enero, 2009). Chlorophenol. [Base de datos]. Recuperado de https://toxnet.nlm.nih. gov/cgi-bin/sis/search2/f?./temp/ KsKwG9:1

TOXNET (21 de enero, 2010). Dimethyl sulphide. [Base de datos]. Recuperado de https:// toxnet.nlm.nih.gov/cgi-bin/sis/search2/f?./ temp/ W2bCTf:1

TOXNET (13 mayo, 2010). Ethyl acrylate. [Base de datos]. Recuperado de https://toxnet.nlm.nih. gov/cgi-bin/sis/search2/f?./temp/ eEVfnJ:1

TOXNET (13 de septiembre, 2012). Hydrogen Sulfide. [Base de datos]. Recuperado de https:// toxnet.nlm.nih.gov/cgi-bin/sis/search2/f?./ temp/ jYYkVj:3

TOXNET (09 de abril, 2013). Methyl mercaptan. [Base de datos]. Recuperado de https:// toxnet.nlm.nih.gov/cgi-bin/sis/search2/f?./ temp/ TA2kiC:1

TOXNET (15 mayo, 2014). Benzene. [Base de datos]. Recuperado de https://toxnet.nlm.nih.gov/ cgi-bin/sis/search2/f?./temp/ M5d2ed:1

TOXNET (04 de septiembre, 2014). Styrene. [Base de datos]. Recuperado de https://toxnet.nlm.nih. gov/cgi-bin/sis/search2/f?./temp/ EwnzeK:1

TOXNET (14 mayo, 2015). Acetaldehyde. [Base de datos]. Recuperado de https://toxnet.nlm.nih. gov/cgi-bin/sis/search2/f?./temp/ qgb7AD:1

TOXNET (22 de septiembre, 2016). Ammonia. [Base de datos]. Recuperado de https://toxnet.nlm.nih. gov/cgi-bin/sis/search2/f?./temp/ VctMjq:1

Universidad Politecnica de Valencia, (2008). Guía técnica para la gestión de las emisiones odoríferas generadas por las explotaciones ganaderas intensivas. Valencia: Universidad Politecnica de Valencia.

Xarxa ambiental, (s.f). Guía de tecnologías limpias en el ámbito de olores. Recuperado de https:// www.malosolores.org/pdf/cliente/guia-tecnologias-limpias-olores.pdf

X.-L. Cao and C. N. Hewitt, (1995) Detection methods for the analysis of biogenic non-methane hydrocarbons in air Journal of Chromatography A 710 39-50 
